\title{
Eigenfertigung oder Fremdbezug - Kriterien und Entscheidungsprozeß zur Gestaltung der betrieblichen Leistungstiefe
}

\author{
Prof. Dr: rer. pol. Helga Meyer
}

Make-or-Buy Entscheidungen sind sehr komplexe Entscheidungen, da prinzipiell alle im Unternehmen erstellten Leistungen dieser Prüfung unterzogen werden können und sollten. Zum anderen steht ein breites Spektrum von Entscheidungsalternativen zur Verfügung. Eine oftmals unterstellte Dichotomie in „entweder" Fremdbezug „oder" Eigenfertigung existiert dagegen nicht. Make-or-Buy Entscheidungen haben eine besondere Bedeutung für die Überlebensfähigkeit von Unternehmen. Sie diurfen deshalb nicht nur aufgrund kurzfristiger Datenbasis entschieden werden. Die Gefahr besteht, daß das betroffene Unternehmen innovative Aufgaben zugunsten von einfachen bekannten Aufgaben vernachlässigt. Vor dem Hintergrund einer zunehmend vernetzten Gesellschaft (Informationsnetzwerke, Videokonferenzen) ist davon auszugehen, daß neue innovative Formen der Kooperation entstehen. Insbesondere für kleine und mittelständische Unternehmen wird es zukünftig zum wichtigen Überlebensfaktor, uiber neue mögliche Kooperationsformen gut informiert zu sein. Aber welche Kriterien müssen bei Make-or-Buy Entscheidungen berüicksichtigt werden und wie kann der Entscheidungsprozeß strukturiert werden? Hierzu soll die folgende Diskussion einen Beitrag leisten.

\section{Gegenstand von Make-or-Buy Entscheidungen}

In der Diskussion zu dem Thema „Eigenfertigung oder Fremdbezug“ werden verschiedene Begriffe $\mathrm{z}$. T. synonym verwendet:

1. Make-or-Buy Entscheidungen - Eigenfertigung oder Fremdbezug

2. optimale Leistungs- bzw. Fertigungsstiefe - vertikale Integration

3. Outsourcing

Die Begriffe der ersten und zweiten Gruppe behandeln im Prinzip den gleichen Tatbestand. Vielleicht wird für den einen oder anderen Betrachter die strategische Bedeutung des Themas durch die Begriffe der zweiten Gruppe deutlicher. Der Begriff Outsourcing verdient eine detailliertere Betrachtung. Outsourcing (outside resource using) allgemein definiert, heißt, man bedient sich außerhalb des Unternehmens liegender Quellen. Dies kann sich auf Produkte oder Dienstleistungen beziehen. Nur solche Leistungen, die bereits im Unternehmen durchgeführt werden, können Gegenstand von Outsourcing Maßnahmen werden, denn nur in diesen Fällen können Überlegungen zu einer möglichen Verla- gerung überhaupt angestellt werden. In der Praxis wurde der Begriff Outsourcing anfänglich häufig in Zusammenhang mit der Verlagerung von DV-Dienstleistungen genutzt. In bezug auf die Begriffe der ersten und zweiten Gruppe ist Outsourcing daher als untergeordneter Begriff einzustufen. ${ }^{1}$ Manche Autoren verwenden ihn dagegen auch gleichbedeutend. ${ }^{2}$

Zwei zentrale Fragen beschreiben den Gegenstand von Make-or-Buy Entscheidungen:

1. Welche Aufgaben sollen fremdbezogen bzw. in Eigenleistung erstellt werden?

2. Welche Organisationsform ist für die Aufgabenerstellung zu wählen?

Welche betrieblichen Teilleistungen können Gegenstand von Make-or-Buy Überlegungen sein?

Entscheidungen über die Fertigungstiefe betreffen auf der einen Seite die betrieblichen Grundfunktionen Beschaffung, Produktion und Absatz. Aktivitäten in Richtung auf die Beschaffungsseite zu übernehmen bedeutet Rückwärtsintegration. So können Teilprodukte entweder selbst hergestellt oder über den Markt bezogen werden. Vorwärtsintegration beinhaltet dagegen die Übernahme von Aktivitäten auf der Absatzseite. Für ein Unternehmen ist die Leistungstiefe durch das Ausmaß bestimmt, in dem benachbarte Leistungsstufen, wie z. B. Fertigung und Montage von Vorprodukten, weitergehende Montage zu Endprodukten oder die Schritte bis zur Vermarktung und zum Kundendienst jeweils innerhalb eines Unternehmens erbracht werden. Die vorherrschende Leistungstiefe ist in den einzelnen Branchen durchaus unterschiedlich. Betriebe mit sehr hoher Leistungstiefe finden sich beispielsweise in der Stahlund Chemiebranche. ${ }^{3}$

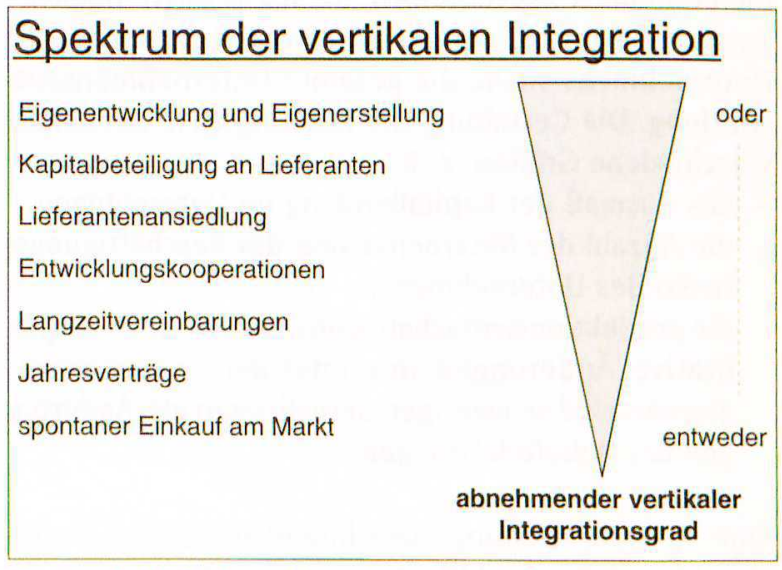

Abb. 1: Beispiele für Entscheidungsalternativen der Fertigungsstufenoptimierung 4 
Auf der anderen Seite können die betrieblichen Querschnittsfunktionen, auch Unterstiitzungsfunktionen genannt, wie Organisation, Personalwirtschaft, Rechnungswesen, Forschung und Entwicklung usw. grundsätzlich ebenfalls Gegenstand einer Make-or-Buy Betrachtung sein. Unterstiitzungsfunktionen wie z.B. Reinigung, Fuhrpark oder Beratungsleistungen sind bekannte Beispiele für Leistungen, die Betriebe iiber den Markt beziehen.

\section{Welche Alternativen stehen zur Wahl?}

Die Ausdriicke Eigenfertigung oder Fremdbezug und Make-or-Buy täuschen auf den ersten Blick eine „Entweder-Oder" Situation vor, die so nicht existiert. Vielmehr gibt es eine ganze Reihe von Alternativen, denn zwischen den Gegensätzen Eigenfertigung und spontaner Einkauf am Markt sind vielfältige Mischformen möglich. Die Make-or-Buy Entscheidung bezieht sich damit nicht nur auf die Frage „was“ intern oder extern erstellt werden soll, sondern ebenso auf die Gestaltung der Beziehungen zu Lieferanten und Kunden, also z. B. auf die Festlegung der Kooperations- und Vertragsformen. Verschiedene Alternativen für die Kooperationsbeziehungen sind denkbar, z. B. Jahresverträge, Entwicklungskooperationen, Lieferantenansiedlung, Joint ventures, Franchise-Verträge, Rahmenlieferverträge.

Der Umstand, daß es sich gerade nicht um eine „Entweder-Oder" Alternative handelt, erhöht die Komplexität des Entscheidungsprozesses erheblich. Vor dem Hintergrund einer zunehmend vernetzten Gesellschaft (Informationsnetzwerke, Videokonferenzen) ist außerdem davon auszugehen, daß neue innovative Formen der Kooperation entstehen. Insbesondere für kleine und mittelständische Unternehmen wird es zukünftig zum wichtigen Überlebensfaktor, iiber mögliche Kooperationsformen gut informiert zu sein.

\section{Bedeutung und Anlässe von Make-or-Buy Entscheidungen}

Bei den verschiedensten Anlässen ist die Unternehmensführung gefragt, über Eigenfertigung oder Fremdbezug zu entscheiden. In den letzten Jahren hat vor allem die strategische Bedeutung der Leistungstiefe für Unternehmen erheblich zugenommen. Sie hat positive oder negative Wirkungen auf die Wettbewerbsposition des Unternehmens sowie die gesamte Unternehmensentwicklung. Die Gestaltung der Leistungstiefe beeinflußt verschiedene Größen, z. B.

- das Ausmaß der Kapitalbindung im Unternehmen,

- die Anzahl der Mitarbeiter und das Beschäftigungsrisiko des Unternehmens,

- die produktionswirtschaftliche Flexibilität, denn qualitative Änderungen des internen Leistungsprogramms sind schwieriger zu realisieren als Änderungen der Zulieferleistungen.

Eine zu hohe Leistungstiefe bindet unnötig Managementkapazitäten und Kapital, die dann für die eigentlichen Kernaufgaben des Unternehmens nicht mehr zur
Verfuigung stehen. Führungsaufmerksamkeit wird in den Binnenbereich gelenkt. In der heutigen Zeit ist jedoch mehr denn je Sensibilität für Umfeldentwicklungen gefragt. Es besteht die Gefahr, daß einfache Aufgaben intern gepflegt werden, während zukunftsträchtige, innovative Aufgaben anderen überlassen werden.

Die folgenden Schlagworte verhelfen dem Aspekt Makeor-Buy zu einer ,neuen“ Aktualität ${ }^{5}$ :

- Konzentration auf das Kerngeschäft,

- Bildung uiberschaubarer Einheiten,

- Höhe und Struktur der Kosten,

- Flexibilität des Unternehmens,

- Globalisierung des Wettbewerbs - aufgrund des technischen Aufholprozesses der Niedriglohn-Länder können heute Hochtechnologie Leistungen qualitativ ausreichend auf dem Weltmarkt beschafft werden,

- günstigere Transaktionsbedingungen, z. B. durch Just-in-time,

- Wettbewerbsvorteile wurden z. B. in der Automobilindustrie durch Verkürzung der Fertigungstiefe erzielt.

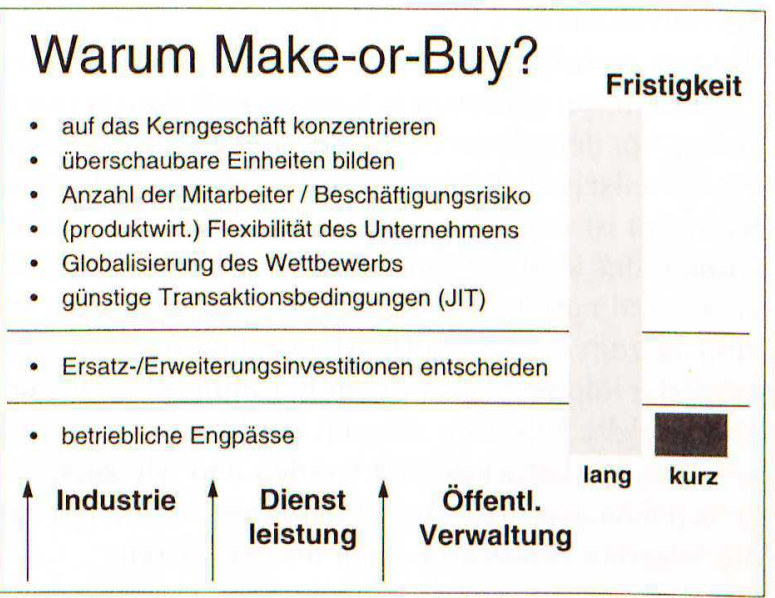

Abb. 2: Anlässe für Make-or-Buy Entscheidungen

Make-or-Buy Fragen können auch aus anderen konkreten Entscheidungsproblemen entstehen. Eine Entscheidung uiber Ersatz- oder Erweiterungsinvestitionen ist zu treffen. Unter Umständen kann es sein, daß sich die Entscheidungsträger gar nicht der Tragweite ihrer Entscheidung bewußt sind. Dann nämlich, wenn sie die Perspektive Make-or-Buy gar nicht in den Entscheidungsprozeß integrieren. Weiterer Handlungsbedarf entsteht beispielsweise im Rahmen der praktischen Produktionsprogrammplanung, wenn sich herausstellt, daß die existierenden Kapazitäten nicht ausreichen.

Der letztgenannte Anlaß beschreibt die kurzfristig notwendige Wahl zwischen Eigenfertigung und Fremdbezug bei Vorliegen eines betrieblichen Engpasses. Die erstgenannten Fragen dokumentieren dagegen ausweislich die strategische Bedeutung der Make-or-Buy Fragestellung. ${ }^{6}$

Make-or-Buy Entscheidungen sind nicht nur für Industriebetriebe von großem Interesse. Auch Dienstleistungsbetriebe müssen zwischen Eigen- und Fremdleistung wählen. Im Zuge knapper Haushaltsmittel ist diese Frage längst auch interessant für die öffentliche Verwaltung. So untersucht beispielsweise die Stadtverwaltung Köln in Folge eines Ratsbeschlusses Aufgaben- 
bereiche und Aufgabenteile der Stadtverwaltung dahingehend, ob ein externer Anbieter bestimmte Leistungen besser, schneller oder kostenguinstiger erbringen kann. ${ }^{7}$

\section{Kriterien für die Gestaltung der Fertigungstiefe}

Die Auswirkungen von Make-or-Buy Entscheidungen sind sehr vielfältig. Die Entscheidungsfindung muß deshalb diverse Kriterien beruicksichtigen. Zwei zentrale Betrachtungsperspektiven existieren, die Produktionskosten und die Transaktionskosten.

\section{Produktionskostenansatz}

Aus Sicht kostenrechnerischer Ansätze werden die Fremdbezugskosten, sprich Marktpreise, verglichen mit den entscheidungsrelevanten Kosten der Eigenfertigung. Denn durch Reduktion der Fertigungstiefe werden variable und teilweise auch fixe Produktionskosten auf Seiten des Abnehmers eingespart. Es entstehen neue Kosten fuir die Beschaffung. Sinken die Beschaffungskosten unter die Produktionskosten, so ergibt sich ein Kostenvorteil.

Welche Kriterien müssen bei der Ermittlung entscheidungsrelevanter Kosten berïcksichtigt werden? ${ }^{8}$

\section{Auslastung der Eigenfertigungsbereiche}

Existiert Unterbeschäftigung, sind nur die zusätzlichen variablen Kosten, z. B. einmalige Kosten für das Reinigen, Umrüsten der Maschinen, Sonderkosten für Werkzeuge und Vorrichtungen, Materialaufwendungen, Aufwendungen für Hilfsstoffe, Energien sowie ggf. Standardsätze für die Abnutzung verschleißender Betriebsmittel zu beriicksichtigen. Unberïcksichtigt bleiben dagegen fixe Bereitschaftskosten, wie Raumkosten, zeitbedingte Abschreibungen für Maschinen, Fertigungslöhne, die zwar langfristig variabel, kurzfristig jedoch fix sind.

Herrscht Vollbeschäftigung und sind die oben ermittelten Kosten ohnehin höher als Fremdbezug, dann ist die Fremdvergabe sinnvoll. Stellt sich dieser Vergleich zunächst zugunsten der Eigenfertigung dar, so müssen die Opportunitätskosten in die Betrachtung einbezogen werden. Das bedeutet, es muß ermittelt werden, wie die benötigten Kapazitäten bereitgestellt werden können, welche zusätzlichen Kosten dadurch entstehen und ob Ertragseinbußen in Kauf genommen werden müssen.

\section{Fristigkeit der Entscheidung}

Bei langfristigen Entscheidungen spielen außerdem kurzfristig fixe Kosten, z. B. für den Auf- bzw. Abbau von Kapazitäten eine Rolle. Zudem müssen die Gesamtkosten beider Alternativen für alternative Bedarfsmengen sowie Investitionsausgaben und eventuell zusätzlich erforderliche Gemeinkostenpotentiale berüicksichtigt werden.

Kooperationsbeziehung zwischen Abnehmer und Lieferant Die Gestaltung der Mischformen, die zwischen den bei- den Extremen Eigenfertigung und Fremdbezug liegen, bietet eine zusätzliche Möglichkeit, die Kosten der Leistungserstellung beim Lieferanten zu reduzieren. Durch Bezugsbindungen kann der Lieferant seine Produktionsmenge vervielfachen, Skaleneffekte (economies of large scale production) und Lernkurveneffekte können dadurch erzielt werden. Diese Vorteile können jedoch auch bei Eigenfertigung genutzt werden.

Lohnkostenvorteile entstehen durch Zugehörigkeit zu unterschiedlichen Branchen. Die Vereinbarung von Wettbewerbsklauseln, bietet dem Zulieferer die Möglichkeit Mengenvorteile zu nutzen, in dem er zusätzlich andere Wettbewerber des Abnehmers beliefert. Sofern der Zulieferer in Fertigungskapazitäten investieren muß, hat die Vertragslaufzeit Auswirkungen auf die Durchsetzung von Produktionskostenvorteilen.

Die verschiedenen Kooperationsbeziehungen als Kriterium für die Ermittlung entscheidungsrelevanter Kosten erhöhen die Komplexität der Entscheidungssituation beträchtlich. Der Produktionskostenansatz unterstellt dagegen eine Dichotomie der Entscheidungsituation: entweder wird ein z. B. vorgelagerter Fertigungsprozeß vertikal integriert (make-Entscheidung) oder er wird vertikal desintegriert (buy-Entscheidung). Über die Gestaltung der Fremdbezugsprozesse, d. h. wie die Beziehungen zwischen Abnehmer und Zulieferer gestaltet werden können, kann keine Aussage getroffen werden. ${ }^{9}$

Weitere Probleme ergeben sich in der Praxis der Entscheidungsfindung:

Objektive Kostendaten: Die objektive und sachgerechte Feststellung der Eigenerstellungskosten wird oft durch divergierende Abteilungsinteressen behindert. Das Ergebnis steht schon vorher fest und wird mit Kostendaten „objektiviert".

Die Schwierigkeit des Vergleichspreises: Lieferanten bieten u. U. unter Selbstkosten an. Die Anfangsverluste werden nach Vertragsschluß durch überhöhte Kosten für Änderungen des Bestellers kompensiert. Informationsvorteile werden vom Lieferanten ausgenutzt, eine ex ante Standardsituation wird zu einer ex post spezifischen Beziehung.

Nicht monetäre Einflußgrößen: Qualitative Kriterien wie Flexibilität, Abhängigkeit, werden nicht erfaßt; der Ansatz konzentriert sich auf Produktionskosten.

Praxis der Entscheidungsfindung: In der Praxis werden häufig längerfristige Entscheidungen auf Basis kurzfristiger Daten gefällt. Wegen der falschen Kostenbasis fuihrt dies zur systematischen Bevorzugung der Eigenerstellung. Der Eigenerstellunganteil steigt an, bei zunehmender Automatisierung der Eigenfertigungsbereiche und sukzessiven Investitionsentscheidungen wird dies noch verstärkt.

\section{Transaktionskostenansatz}

Transaktionskosten werden auch als „Betriebskosten des Wirtschaftssystems" bezeichnet. Sie umschreiben das ökonomische Gegenstiick zur Reibung in der Physik. Mit den Transaktionskosten wird die vertikale Integration zum Problem der Vertragstheorie. ${ }^{10}$ Transaktionen sind Tauschaktivitäten. Unter Transaktionskosten 
versteht man demgemäß die Kosten der Information und Kommunikation, die bei der Organisation und $\mathrm{Ab}$ wicklung arbeitsteiliger Leistungserstellung anfallen. ${ }^{11}$

\begin{tabular}{|l|l|}
\hline \multicolumn{2}{|c|}{ Transaktionskosten - Beispiele } \\
\hline $\begin{array}{l}\text { - } \begin{array}{l}\text { Suchkosten } \\
\text { - Informations-/Verhandlungskosten }\end{array} \\
\text { - Tauschkosten }\end{array}$ & ex ante \\
\hline $\begin{array}{l}\text { - } \text { Konsicherungskosten Vellkosten } \\
\text { Verhandlung nachträglicher Anpassung } \\
\text { - durch eigene Fehlplanung } \\
\text { - durch opportunistisches Verhalten } \\
\text { - nicht antizipierte Umweltveränderungen }\end{array}$ & \\
\hline
\end{tabular}

Abb. 3: Transaktionskostenarten ${ }^{12}$

Transaktionskosten entstehen nicht nur zwischen Organisationen, sondern auch in Organisationen. Durch die Integration von Aktivitäten in die Unternehmung entstehen Kosten, z.B. für Verwaltung, Koordination und Kontrolle. Die Transaktionskosten können in zwei Gruppen untergliedert werden, die ex ante und ex post Transaktionskosten. Je höher die ex ante Transaktionskosten, im Extremfall die Integration der Leistung, desto geringer sind die ex post Transaktionskosten.

Die Höhe und Struktur der Transaktionskosten ist davon abhängig, in welcher Weise die arbeitsteilige Leistung institutionell verankert ist. Institutionelle Verankerung umfaßt die Organisations- oder Vertragsform, also auch die Frage, ob es sich um Eigenfertigung oder Fremdbezug handelt. Die Eigenschaften der Leistung, des Transaktionsobjektes, bilden die zweite Einflußgröße. Die Zusammensetzung und Höhe der Transaktionskosten hängt damit von zwei Dingen ab:

- den Eigenschaften der jeweiligen Leistung,

- der institutionellen Einbindungsform des Transaktionsobjektes.

Die Entscheidungsfindung für Make-or-Buy anhand des Transaktionskostenansatzes versucht deshalb, die jeweiligen Leistungsarten, die ein Unternehmen für seine marktliche Aufgabe benötigt, derart mit den verfügbaren institutionellen Verankerungen zu kombinieren, daß die Transaktionskosten insgesamt minimiert werden.

\section{Eigenschaftsmerkmale des Transaktionsobjektes}

Folgende Eigenschaften sind fuir die Aussage zu den Transaktionskosten relevant

- Spezifität,

- Strategische Bedeutung und

- als sekundäre Größen Unsicherheit und Häufigkeit.

Spezifität: Mit zunehmender Spezifität erhöhen sich die gegenseitigen Abhängigkeiten und Sicherungsbeduirfnisse. Deshalb ist ein stabiler und integrativer Rahmen etwa in Form langfristiger Verträge oder gar Eigenfertigung erforderlich. In der Industrie ist dieses Merkmal typisch fuir Anlagen oder Werkzeuge. Spezifität kann sich aber ebenso auf Personalqualifikationen, Logistik,
Fertigungsverfahren oder Qualitätseigenschaften beziehen und steht im Gegensatz zur standardisierten Leistung.

Strategische Bedeutung: Strategisch bedeutsame Leistungen sind in der Regel gleichzeitig ausgesprochen unternehmenspezifische Leistungen, da sich die Unternehmen mit diesen Leistungen ganz gezielt von anderen Wettbewerbern differenzieren wollen. Strategisch bedeutsame Leistungen müssen geheimgehalten werden. Dies ist in der Regel intern leichter möglich. Fremdbezug wuirde zusätzlich erhebliche Überwachungs- und Kontrollkosten verursachen.

Unsicherheit und Häufigkeit: Unsicherheiten fuihren zu nachvertraglichen Anpassungen. Nur wenn es sich um eine standardisierte und strategisch nicht so bedeutsame Leistung handelt, kann sie effizient über marktliche Verträge, etwa durch Rahmenvereinbarungen mit flexiblen Abrufmengen eingebunden werden. Je häufiger eine spezifische, strategisch bedeutsame Leistung erstellt werden muß, desto sinnvoller die Tendenz zur vertikalen Integration.

Zusammenfassend können zwei grundlegende Strategieempfehlungen abgeleitet werden ${ }^{13}$ :

1. Eigenfertigung bringt dem Unternehmen Produktions- und Transaktionskostennachteile, wenn die zu erbringenden Leistungen gut strukturiert, standardisiert, sicher und von geringer strategischer Bedeutung sind,

2. Eigenfertigung bringt dem Unternehmen einen erheblichen Transaktionskostenvorteil, wenn die zu erbringenden Leistungen strategisch wichtig, unternehmenspezifisch sowie unsicher sind und außerdem noch häufig anfallen.

Es gilt jedoch zu prüfen, ob das unternehmensinterne Know-how ausreicht. Ist das unternehmensexterne Know-how deutlich überlegen, wird eine vertikale Kooperationsform, d. h. Fremdbezug, eher effizient sein.

Institutionelle Einbindungsform des Transaktionsobjektes Die institutionelle Einbindungsform des Transaktionsobjektes ist die zweite entscheidende Einflußgröße der Transaktionskosten. Hierbei geht es hauptsächlich um die Gestaltung der Handlungsalternativen bei vertikaler Desintegration. Beispielsweise muissen Bezugsbindungen, Wettbewerbsklauseln oder Vertragslaufzeiten zunächst ausgehandelt und später kontrolliert werden.

\section{Der Prozeß der Entscheidungsfindung}

Der Entscheidungsprozeß für Make-or-Buy kann anhand des Regelkreismodells der Leitungsfunktionen in verschiedene Phasen und Elemente zerlegt werden. ${ }^{14}$ Das Entscheidungssubjekt, der Regler, muß bei seiner Aufgabenbewältigung gewisse Vorgaben berücksichtigen, beispielsweise die Entwicklung im wirtschaftlichen und gesellschaftlichen Umfeld. Für eine Betrachtung der möglichen Alternativen müssen Informationen zu den möglichen Alternativen eingeholt werden. Erst wenn ausreichend Informationen vorliegen, können die Alternativen einer Bewertung unterzogen werden mit der 
anschließenden Auswahl einer Lösung. Für die Durchfuihrung der gewählten Alternative ist ein geeigneter organisatorischer Rahmen zu schaffen. Sobald es zur Durchführung kommt muissen die Ergebnisse einer regelmäßigen Kontrolle unterzogen werden. Kontrolle bedeutet in diesem Zusammenhang, Istwerte ermitteln und mit den gesetzten Zielen vergleichen. Je nach Differenz zwischen Soll und Ist muß eine Abweichungsanalyse vorgenommen werden. Die Ergebnisse minden in den erneuten Prozeß der Willensbildung. Der Entscheidungsprozeß kann damit als kybernetisches System beschrieben werden, das eine zielgerichtete Lenkung von Prozessen unter Einfluß von Störungen ermöglicht.

Auch der Entscheidungsprozeß zu Make-or-Buy kann im Sinne eines Regelsystems strukturiert werden, indem den beschriebenen Tätigkeitsstufen konkrete Aufgaben des Make-or-Buy Entscheidungsprozesses zugeordnet werden.

\section{In der Phase der Willensbildung}

Innerhalb einer Aufgabenanalyse müssen alle potentiellen Leistungen, die im Unternehmen erbracht werden, der Frage unterzogen werden, ob sie auch extern erstellt werden können. Grundsätzlich kommen für diese Analyse alle Leistungen des Unternehmens in Betracht. Die Aufgaben werden hinsichtlich ihrer Struktur analysiert und bewertet. Hierfuir ist Klarheit dariber herzustellen, welche Arbeitsschritte zur Erstellung der Leistung erforderlich sind. Es entsteht ein Leistungsverzeichnis. Die weitergehende Analyse der Aufgabenstruktur geschieht zweckmäßigerweise anhand der nach dem Transaktionskostenansatz wichtigen Eigenschaftsmerkmale Spezifität, strategische Bedeutung, Häufigkeit und Unsicherheit. Auf der Basis der identifizierten Teilarbeitsschritte müssen die Kosten für Eigenfertigung und Fremdbezug ermittelt werden. Intern muissen dazu die entsprechenden Kostendaten von den jeweiligen Stellen erhoben werden. Die möglichen Kosten für externe Beschaffung können über eventuell vorliegende Preisspiegel, Erfahrungswerte oder Einholen von Angeboten ermittelt werden.

Als Basis für die Auswahl der möglichen Alternativen müssen demnach folgende Ergebnisse vorliegen: Ko-

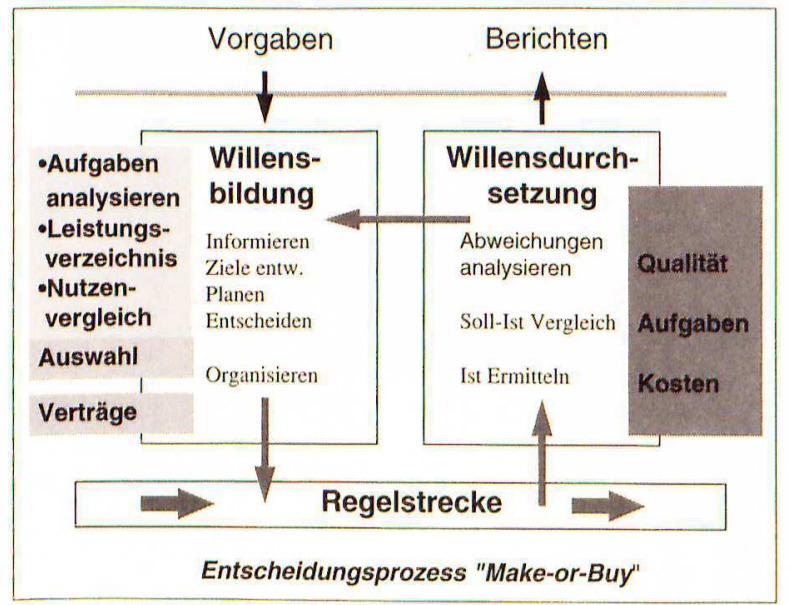

Abb. 4 : Der Entscheidungsprozeß „Make-or-Buy“ anhand des Regelkreismodells der Leitungsfunktionen stenvergleichsrechnungen für die Produktionskosten und eine Bewertung der zu erwartenden Transaktionskosten. Zusätzliche Entscheidungshilfe kann eine Nutzwertanalyse bieten, die es erlaubt, monetär nicht meßbare Faktoren zu berïcksichtigen. Mit Hilfe der Regelkreistätigkeiten Informieren, Ziele setzen, Planen und Entscheiden kommt es zu einer Filterfunktion, in deren Verlauf das Spektrum der möglichen Handlungsalternativen zu Make-or-Buy immer weiter eingeengt wird. Für den Einsatz in der betrieblichen Praxis wird es sinnvoll sein, betriebspsezifisch standardisierte Strukturierungshilfen, z. B. in Form von Checklisten, zur Verfügung zu stellen.

Fällt die Wahl auf einen externen Lieferanten, müssen für die konkrete Erstellung der Leistung die entsprechenden Rahmenbedingungen geschaffen werde. Hierzu zählen die vertraglichen Vereinbarungen.

\section{In der Phase der Willensdurchsetzung}

Der Entscheidungsprozeß zu Make-or-Buy schließt nach der Entscheidung nicht ab. Periodisch müssen die Auswirkungen der gewählten Alternative hinsichtlich Leistung, Qualität und Kosten regelmäßig immer wieder überprüft werden und einer neuen Make-or-Buy Betrachtung anhand der aufgezeigten Schritte unterzogen werden. Hiermit schließt sich der Regelkreis. Außerdem ist zu beachten, daß der hier beschriebene Regelkreis zu Make-or-Buy keinesfalls ein geschlossenes System ist. Einfluisse von außen, z. B. veränderte Marktsituationen, muissen in den regelmäßig zu wiederholenden Make-or-Buy Betrachtungen entsprechend berïksichtigt werden.

Für die betriebliche Praxis enstehen folgende organisatorische Kernfragen bei der Realisierung eines erfolgreichen Make-or-Buy Entscheidungsprozesses:

- Welchen Kriterien und Regeln folgt die oben beschriebene Filterfunktion?

- Wie kann der Entscheidungsprozeß institutionell so im Unternehmen verankert werden, daß ein kontinuierlicher Regelungsprozeß gewährleistet ist?

\section{Zusammenfassung und offene Fragen}

Bei der Entscheidungsfindung sollten sowohl Kriterien des Produktionskostenansatzes aber vor allem auch die Transaktionskosten berücksichtigt werden. Der Entscheidungsprozeß selbst ist im eigentlichen Sinne nie beendet sondern beschreibt einen kontinuierlichen Regelkreis. Für eine effektive Gewährleistung der beschriebenen Aufgaben sind zuständige Aufgabenträger zu benennen. Eine interdisziplinäre Zusammenarbeit scheint unerläßlich, damit nicht einseitig Kostenbetrachtungen oder technisches Know-how die Entscheidungen beeinflussen. Der anhand des Regelkreismodells vereinfacht dargestellte Entscheidungsprozeß sollte in der Praxis in mehreren Stufen organisiert sein. Interdisziplinäre Teams, die Bildung von Entscheidungsgremien sowie die Zuordnung von spezifischen Aufgaben zu definierten Aufgabenträgern sind zu diesem Zweck zu realisieren.

Weitere interessante Fragestellungen zu dem Thema 
Eigenfertigung oder Fremdbezug ergeben sich aus der zunehmenden Vernetzung der Gesellschaft. Zukünftig werden dadurch sicher neue Kooperationsformen entstehen. Ebenso sind Auswirkungen auf die Höhe der Transaktionskosten denkbar. Die geschilderte Komplexität der Entscheidungssituation gilt es zu reduzieren, ohne daß hierdurch wichtige Informationen aus dem Bewertungsprozeß herausfallen. Hierfür könnten entscheidungsunterstiitzende Systeme eine Hilfe sein.

\section{Anmerkungen}

1 Bliesener, M.-M.: Outsourcing als mögliche Strategie zur Kostensenkung, in: BFuP 4/94, S. 277-290

2 Stadt Köln: Wirtschaftlichkeitsvergleiche zwischen internen und externen Leistungen, o.D.

3 Picot, A.: Ein neuer Ansatz zur Gestaltung der Leistungstiefe, in: zfbf 43 (4/1991), S. 336-357

4 Heinen, E.: Industriebetriebslehre, 9. erw. Auflage, Wiesbaden 1991, S. 423, Benkenstein, M./Henke, N.: Der Grad vertikaler Integration als strategisches Entscheidungsproblem, S. 86

5 Knolmayer, G.: Zur Berücksichtigung von Transaktions- und Koordinationskosten in Entscheidungsmodellen für Make-or-BuyProbleme, in: BFuP 4/1994, S. 316-332

6 Männel, W.: Entscheidungsorientierte Kostenvergleichsrechnungen für den kurzfristigen Übergang von der Eigenfertigung zum Fremdbezug, in Kostenrechnungspraxis 3/1990, S. 187-190

7 Stadt Köln a. a. 0.

8 Männel, W.: Wenn Sie zwischen Eigenfertigung oder Fremdbezug entscheiden müssen..., in: Management-Zeitschrift io 52 (1983) Nr. 7/8, S. 301-307

9 Benkenstein, M.: Die Gestaltung der Fertigungstiefe als wettbewerbsstrategisches Entscheidungsproblem - Eine Analyse aus transaktions- und produktionskostentheoretischer Sicht, in zfbf 46 (6/1994), S. 483-498

10 Williamson, O. E.: Die ökonomischen Institutionen des Kapitalismus, Tübingen 1990

11 Heinen a. a. O.

12 Benkenstein, M./Henke, N. a. a. O., S. 80, und Picot a. a. O., S. 344

13 Heinen a. a. 0.

14 Dworatschek, S. /Meyer, H.: Unification and Professionalization of the Role of Project Personal - empirical data and recomendation on the functions of team members, in: Proceedings of the IPMA Symposium „Modern Project Management: Unification of Professionals for Individual Success", St. Petersburg September 1995, pp. $81-88$

\section{Verfasser}

Prof. Dr. rer. pol. Helga Meyer

Technische Fachhochschule Wildau

Fachbereich Betriebswirtschaft/Wirtschaftsinformatik Tel. (0 33 75) 507-119 und -124 